\title{
El multilateralismo como intervencionismo. Estados Unidos y la Sociedad de Naciones en América Latina (1930-1946)
}

\author{
José Antonio SÁnchez RomÁN \\ Universidad Complutense de Madrid \\ sanchezroman@ccinf.ucm.es
}

Recepción: 12 de enero de 2015 / Revisión: 28 de marzo de 2015

Aceptación: 13 de abril de 2015 / Publicación: Diciembre de 2015

\begin{abstract}
RESUMEN
Este artículo analiza una dinámica de intervenciones de Estados Unidos en América Latina que no ha atraído suficientemente la atención de los historiadores. En los años treinta y cuarenta, cuando Europa se hundía en una nueva confrontación bélica, ciertos sectores del gobierno y del mundo empresarial norteamericano intentaron articular una nueva relación con los países del continente basada en una propuesta de multilateralismo que se había configurado dentro de la Sociedad de Naciones (SN). Estos estadounidenses intentaron establecer una dinámica de relaciones triangulares con los gobiernos latinoamericanos y los organismos técnicos de la SN. Gracias a ello, como se mostrará en este artículo para el caso del funcionamiento del Comité Fiscal de la Sociedad de Naciones, los latinoamericanos fueron capaces de influir en el tipo de políticas que debían emanar de esta relación triangular. La importancia de esta historia no es menor. La relación triangular entre Estados Unidos, América Latina y la SN sirvió de base para la reconstrucción de la gobernanza global liderada por los Estados Unidos tras la guerra.
\end{abstract}

Palabras clave: Relaciones Estados Unidos-América Latina, Sociedad de Naciones, Fiscalidad Internacional, Gobierno Mundial, Estados Unidos, siglo XX.

\section{Multilateralism as Interventionism. United States and the Society of Nations in Latin America (1930-1946)}

\begin{abstract}
This article analyzes a dynamic of the United States' interventions in Latin America, that hasn't sufficiently attracted the attention of historians. In the 1930's and 1940's, when Europe was descending into a new military conflict, certain United States government and business sectors attempted to articulate a new relationship with the nations of the continent, based on a proposal of multilateralism that had been set up within the Society of Nations (SN). These Americans attempted to establish a dynamic of triangular relations with the governments of Latin America and the technical organisms of the SN. As the article will demonstrate in the case of the Society of Nations' Fiscal Committee, thanks to this, Latin-Americans were capable of influencing the type of policies that were to come out of this triangular relationship. The importance of this history is no small matter. The triangular relationship between the United States, Latin America and the SN served as a base for the reconstruction of global governance headed by the United States after the war.
\end{abstract}


Keywords: United States-Latin American Relations, Society of Nations, International Taxation, World Government, United States, $20^{\text {th }}$ Century.

Sumario: 1. Introducción. 2. Multilateralismo y panamericanismo. 3. La cuestión fiscal. 4. Balance: los límites del panamericanismo. 5. Referencias bibliográficas.

\section{INTRODUCCIÓN}

La historia de las intervenciones de Estados Unidos en América Latina en el siglo XX está marcada por la extensión de la doctrina Monroe a través del corolario Roosevelt y por la agresiva política de control de lo que consideraba su esfera "natural" de influencia durante la guerra fría. De hecho, muchos historiadores han subrayado la continuidad entre las intervenciones (a veces militares) de comienzos de siglo y las llevadas a cabo durante la guerra fría, como parte de un proyecto hegemónico de los Estados Unidos hacia el hemisferio occidental que se habría forjado en el siglo XIX y como reacción frente a la dinámica revolucionaria que desde comienzos del siglo XX se desarrolló en América Latina ${ }^{1}$.

En este artículo se explora una dinámica de intervenciones de Estados Unidos en América Latina distinta, que no ha atraído suficientemente la atención de los historiadores. En los años treinta y cuarenta, cuando Europa se hundía en una nueva confrontación bélica, ciertos sectores del gobierno y del mundo empresarial norteamericano intentaron articular una nueva relación con los países del continente basada en una propuesta de multilateralismo que se había configurado dentro de la Sociedad de Naciones ( $\mathrm{SN}$ ). Estos estadounidenses intentaron establecer una dinámica de relaciones triangulares con los gobiernos latinoamericanos y los organismos técnicos de la $\mathrm{SN}$. Por tanto, hegemonía regional y multilateralismo convergieron en este período. Esta forma de intervencionismo no sólo era diferente a la anterior en su cara más amable, siguiendo la línea planteada por la política del Buen Vecino de Roosevelt. Implicaba también una visión del orden global y no sólo una aspiración hegemónica en el hemisferio occidental, es decir, suponía la concreción de los presupuestos wilsonianios, de moldear el gobierno del mundo tomando como patrón el modelo estadounidense. Al mismo tiempo, como se mostrará en este artículo para el caso de las cuestiones fiscales, los latinoamericanos fueron capaces de influir en el tipo de políticas que debían emanar de esta relación triangular. La importancia de esta historia no es menor. La relación triangular entre Estados Unidos, América Latina y la SN era vista por las elites estadounidenses que las impulsaron como parte de un ensayo amplio que les permitiera liderar la reforma de una gobernabilidad global tras el conflicto. En cierta manera, puede decirse que la "reinvención" de la Sociedad de Naciones y la creación del nuevo orden internacional de la segunda posguerra comenzó a forjarse en América Latina.

1 Ver Grandin - Joseph, 2010 y Joseph, 2008. Una visión más atenta a las rupturas en PettinÀ, 2011. 


\section{MULTILATERALISMO Y PANAMERICANISMO}

A pesar de que la crisis de 1929 y el ascenso de los fascismos en Europa parecía haber hecho colapsar los mecanismos multilaterales diseñados por la $\mathrm{SN}$, la propuesta multilateral siguió viva entre los técnicos de la Liga y entre muchos liberales estadounidenses que veían en un régimen multilateral la solución a los problemas de competencia imperial e ineficacia económica internacional. Si esta visión positiva sobre el multilateralismo pervivió fue porque, a pesar del nacionalismo agresivo y del cierre de fronteras en respuesta a la crisis, la idea de que el mundo era inevitablemente "interdependiente" había ganado terreno desde el período de acelerada globalización de finales del siglo XIX y se había convertido en una especie de ortodoxia dentro de la Sociedad ${ }^{2}$.

Pero la visión de la interdependencia global que defendió la SN a partir de la década de 1930 divergía notablemente de una nostalgia por el mundo de finales del siglo XIX. Cuando la Sociedad de Naciones se creó sus objetivos eran claramente europeos y centrados en la cuestión de la recuperación, la estabilidad financiera y la solución al caos monetario. Ideas tan ambiciosas como las de la cooperación internacional y la inclusión de los temas sociales (de salud, alimentación, etc.) que aparecieron a partir de la década de 1930 en la dimensión económica formaron parte de lo que Patricia Clavin ha denominado la reinvención de la Sociedad ${ }^{3}$. Esa reinvención pasó por un proceso en el cual el centro de atención de los economistas liberales de la SN se movió de la recuperación del viejo mundo de la Belle Époque a un planteamiento centrado en las diferencias entre países ricos y países pobres y el problema del nivel de vida del campesinado ${ }^{4}$. El objetivo era salvar el capitalismo y, al mismo tiempo, transformarlo profundamente.

Los técnicos y burócratas de la SN recurrían frecuentemente al concepto de interdependencia, sobre todo a partir de la década de 1930. Había razones múltiples para ello. En primer lugar, se trataba de una cuestión de supervivencia. La depresión económica y posteriormente el colapso del sistema de seguridad colectiva pusieron

2 Ver League of Nations, 1939, p. 7. Los historiadores económicos han sostenido que con la Primera Guerra Mundial llegó a su fin un período de rápida globalización, ver O’RouRKE - WiLliamson, 2006. Sin embargo, en ámbitos como la Sociedad de Naciones se observaba que a pesar de la tendencia creciente hacia la autarquía y la creación de bloques regionales causada por la inestabilidad de la década del veinte y sobre todo por la crisis de 1929, las conexiones globales seguían siendo una realidad. La idea de interdependencia ya formaba parte del arsenal discursivo de los abogados internacionalistas de finales del siglo XIX. Ver KoskenNIEMI, 2005. Los historiadores del siglo XX interesados por la cuestión de la interdependencia se han centrado en la crisis de los años setenta y han visto en el concepto de interdependencia un claro antecedente del de globalización. Véanse los ensayos contenidos en Ferguson - MAIER - MANELA - SARgent, 2010, especialmente los trabajos de Maier y Sargent. En los años setenta y ochenta, el concepto se convirtió en una herramienta política de quienes pretendía un orden económico más desregulado y la restricción de las demandas de la sociedad civil sobre el estado de bienestar (como ocurriría después con el concepto de globalización). Sin embargo, desde finales del siglo XIX y claramente en el período de entreguerras el concepto de interdependencia servía para defender un activismo liberal en el plano internacional. En parte, el legado de este concepto puede encontrarse en los movimientos internacionales pro derechos humanos. En todo caso, aún necesitamos una exploración detenida del concepto de interdependencia.

3 Clavin, 2013.

4 Clavin, 2013, capítulo 6. 
en entredicho la función de la $\mathrm{SN}^{5}$. Al enfatizar la noción de interdependencia, los técnicos de la Liga estaban señalando la necesidad de contar con un organismo internacional que articulara la cooperación internacional. En un mundo interdependiente las crisis no se podían resolver de manera autónoma. Era también una respuesta a las soluciones alternativas que se estaban planteando a la Sociedad: la Esfera de Coprosperidad Asiática propuesta por Japón, el internacionalismo comunista soviético y la visión nazi de Europa. En segundo lugar, la idea de interdependencia permitía a los técnicos de la SN justificar su programa de reforma del capitalismo. El ejemplo paradigmático es el de la cuestión del nivel de vida del campesinado. Los especialistas de la Liga argumentaban que sólo incrementando el nivel de vida de los campesinos de la Europa rural y de los países pobres los países industriales podrían vender sus productos. El capitalismo tenía que garantizar la salud, educación y buena alimentación de la mayoría de la humanidad a través de políticas intervencionistas, que la SN concebía en una escala internacional o supranacional.

El proyecto de regulación de la interdependencia articulado por los organismos técnicos de la Liga se configuró en diálogo con otras visiones de la gobernanza global económica y con otro proyecto de rescate del capitalismo, el que procedió de algunos sectores políticos, intelectuales y empresariales de los Estados Unidos. Este diálogo era una necesidad imperiosa para la propia Liga. En primer lugar, el peso de la economía estadounidense en la economía mundial era tal que cualquier proyecto de reorganización a escala global necesitaba de su participación. En segundo lugar, según Europa se adentraba en un nuevo conflicto bélico, Estados Unidos se convirtió en un refugio para las actividades de la Sociedad de Naciones. En 1940, los organismos técnicos de la SN se trasladaron al Institute for Advance Studies de la Universidad de Princeton, gracias a un subsidio de la Fundación Rockefeller (que llevaba ya colaborando con la Liga desde al menos finales de la década de 1920) y con la colaboración del gobierno de los Estados Unidos. Mientras Europa se desangraba en una nueva guerra, la administración Roosevelt y parte de las elites estadounidenses se comprometieron en reorganizar los organismos multilaterales fortaleciendo el sistema de la Sociedad de Naciones.

Pero la aproximación entre la Sociedad de Naciones y Estados Unidos tuvo otro escenario, el de América Latina. El panamericanismo y las políticas que los Estados Unidos habían diseñado para América Latina podían ser concebidos como otra de las alternativas regionales a la visión global de la SN. En algún sentido lo eran. En la década de 1930 muchos países latinoamericanos abandonaron la Sociedad de Naciones, ya fuera movidos por razones económicas o por la percepción de que el organismo internacional estaba en clara decadencia ${ }^{6}$. Muy rápidamente una vinculación más estrecha con los Estados Unidos, ya fuera a través de mecanismos bilaterales o dentro de un renovado sistema panamericano, se ofreció como una alternativa plausible y fructífera para muchos latinoamericanos. Pero esto no cerraba las puertas a la coope-

5 Las sucesivas crisis de seguridad de los años treinta llevaron a la Sociedad a redimensionar sus tareas técnicas, lo que Walters, 1971, p. 717, ha llamado "Renacimiento de las agencias económicas y sociales".

6 FisCHER, 2012. 
ración y a la integración de visiones, que aunque pudieran ser contrapuestas encontraron territorio para la coincidencia en un momento de profunda crisis.

Desde principios de siglo XX las relaciones entre Estados Unidos y América Latina habían estado marcadas por la desconfianza. La agresiva política estadounidense en el Caribe y la no menos agresiva acción económica había marcado la imagen del país del norte para muchos latinoamericanos ${ }^{7}$. Pero la relación entre los vecinos adquirió rasgos más complejos a partir de la década de 1920. La integración tradicional de los países latinoamericanos en los mercados mundiales a través de la exportación de bienes primarios empezó a verse cuestionada. En la década de 1920 los términos de intercambio latinoamericanos se deterioraron o se volvieron muy inestables ${ }^{8}$. Este proceso se acompañó de una modificación en las relaciones comerciales latinoamericanas. Estados Unidos se convirtió en el principal socio comercial de la región, significando el 34\% de las exportaciones en 1929 y el 38\% de las importaciones ${ }^{9}$.

Hasta la Primera Guerra Mundial América Latina había recibido una sustancial cantidad de capital europeo a través de inversiones en cartera y préstamos al sector público. A partir del conflicto, los lazos de la región con los mercados de capital internacionales se transformaron. En primer lugar, las inversiones europeas se frenaron en la década de 1920 y las inversiones norteamericanas se multiplicaron. De 1913 a 1926, las inversiones estadounidenses en América Latina pasaron de 1.276 millones de dólares a 5.370. Mientras en 1913, esas inversiones representaban el 18\% del total de las inversiones extranjeras en la región, en 1926 ya suponían el 48\%. Además, el crecimiento de la presencia financiera estadounidense en la región significó también una modificación cualitativa en las inversiones. Es cierto que las inversiones en cartera estadounidenses, en proporción, crecieron durante la década de 1920 gracias al papel de Wall Street como mercado para la venta de valores latinoamericanos. Pero las inversiones directas estadounidenses aún significaban el $68 \%$ del total de inversiones de ese país en la región en $1929^{10}$. Esta estructura de inversiones era diferente a las tradicionales europeas que habían tenido su período de esplendor antes de la guerra. Esta novedad estaba indicando ya la importancia que las multinacionales estadounidenses estaban adquiriendo en muchos países de América Latina.

El crecimiento de las relaciones comerciales y, sobre todo, financieras entre Estados Unidos y América Latina en la década de 1920 adquirió en algunos casos dimensiones febriles, acompañadas de episodios de corrupción y sobornos. En Colombia el fenómeno fue bautizado como la "danza de los millones". Pero la llegada del dinero estadounidense también se acompañó, en el caso de las naciones andinas, de importantes novedades institucionales. En la década de 1920, Colombia, Ecuador, Perú, Bolivia y Chile recibieron la visita de Edwin Kemmerer, un economista norteamericano experto en cuestiones financieras y fiscales. Las llamadas misiones Kemmerer tenían como objetivo aconsejar a los gobiernos andinos sobre las reformas fiscales y

7 La bibliografía es muy vasta. Además de la introducción y los primeros tres capítulos de GRANDIN - JOSEPH, 2010, se pueden consultar, entre otros, RosenberG, 1987; Tulchin, 1971; o HugGins, 1998.

8 Bulmer-Thomas, 1994, pp. 162-174.

9 Bulmer-Thomas, 1994, p. 159.

10 Bértola - OCAMPo, 2013, pp. 135-137. 
financieras necesarias con el objetivo de estabilizar las cuentas públicas y permitir a estos países (re)ingresar al patrón oro.

Este programa de reforma era visto como imprescindible por los prestamistas estadounidenses antes de aprobar programas de financiación de los gobiernos andinos. Estos requerían la presencia de Kemmerer en buena medida como una forma de tranquilizar a sus posibles prestamistas en Estados Unidos. Pero las misiones Kemmerer produjeron resultados notables y en gran manera inesperados. Las repúblicas andinas acentuaron su dependencia de la economía estadounidense, pero al mismo tiempo, gracias a los instrumentos creados por las propuestas de Kemmerer, como los bancos centrales o los impuestos a la renta, los gobiernos desarrollaron nuevas capacidades para intervenir en sus economías. Por tanto, las misiones Kemmerer no sólo establecieron las bases para una política comercial liberal (open door policy) y relaciones más estrechas comerciales y financieras con los Estados Unidos, sino también crearon mecanismos que permitirían a los gobiernos andinos proteger sus economías cuando la gran depresión hiciera notar sus efectos ${ }^{11}$.

La creciente interdependencia entre Estados Unidos y América Latina estaba marcada por la complejidad. Sería un error pensar que las repúblicas andinas adoptaron a rajatabla las recomendaciones de Kemmerer y que esto condujo inexorablemente a una mayor subordinación a los Estados Unidos. De hecho, como ha señalado Paul Drake, muchas de las reformas propuestas por Kemmer ya habían sido discutidas por las elites locales y la visita del economista estadounidense era un mecanismo para reforzar la legitimidad de estas políticas. Un caso interesante es el de los bancos centrales. En muchos casos, Kemmerer se limitó a discutir proyectos que ya habían sido elaborados por los gobiernos andinos. Estos proyectos, a su vez, habían sido diseñados bajo la influencia de las conferencias internacionales de Bruselas (1920) y Génova $(1922)^{12}$.

Además, algunas de las recomendaciones de Kemmerer nunca fueron puestas en práctica o cuando lo hicieron se desvirtuó la intención original. De hecho, otros países latinoamericanos aplicaron políticas similares a las diseñadas en los países andinos sin la visita de Kemmerer. En las décadas de 1920 y 1930 los experimentos fiscales y monetarios se repitieron en otros países de la región ${ }^{13}$.

Otro aspecto importante que hay que destacar es que Kemmerer actuaba como un agente privado y no como un representante del gobierno de los Estados Unidos. Los representantes diplomáticos de Washington en Lima, Bogotá o Quito intentaban ofrecer una imagen de distanciamiento con respecto a las actividades de Kemmerer. Pero las misiones del economista ayudaron a construir lazos más estrechos entre las naciones sudamericanas y los Estados Unidos. Las visitas de Kemmerer reproducían la estrategia empleada por Washington en Europa Central, donde buscó influir en las políticas de estabilización a través de la actuación de economistas particulares y evitando implicarse directamente ${ }^{14}$. En 1926, por ejemplo, Kemmerer fue invitado por el gobierno polaco a diseñar un programa de reforma financiera y económica. Como

\footnotetext{
11 Sobre las importantes misiones Kemmerer ver el excelente trabajo de DraKE, 1989.

12 DraKe, 1989, p. 255.

13 Por ejemplo en Brasil o Argentina. Véase Bulmer-Thomas, 1994, pp. 180-181 y Corol, 2011, p. 31.

14 Drake, 1989, p. 267 y Costigliola, 1979, p. 87.
} 
ocurrió en los casos andinos, la asesoría de Kemmerer ayudó a Polonia a acceder a los créditos estadounidenses ${ }^{15}$.

Este proceso revela las complejas interacciones entre Estados Unidos, América Latina y Europa. Kemmerer vivió una experiencia europea contemporánea a su visita a América Latina. Las repúblicas andinas recibieron la influencia de las conferencias financieras internacionales organizadas en Europa antes de la visita de Kemmerer. Y tanto en Polonia como en Perú, las elites locales buscaban la ayuda y la cooperación de los Estados Unidos ${ }^{16}$. Se estaba creando un patrón de relaciones triangulares que sería habitual en las décadas de 1930 y 1940.

Los años veinte fueron años de "americanización" en América Latina. Además de la profundización de las conexiones económicas y financieras, formas de cultura popular y patrones de consumo que suelen asociarse a los Estados Unidos se extendieron al sur del continente. Este proceso había comenzado antes en México o Cuba, pero ahora cobró impulso. Como en el caso de las relaciones financieras, estas nuevas formas de consumo o la irrupción del cine fueron fenómenos complejos que sobrepasan las aspiraciones estadounidenses a la hegemonía cultural y comercial. En muchos casos, las influencias y movimientos no iban simplemente del "centro" (Estados Unidos) a la "periferia" (América Latina), sino que tomaban otros derroteros ${ }^{17}$.

En todo caso, la presencia de Estados Unidos en el hemisferio tenía a finales de la década de 1920 raíces más sólidas que en cualquier momento anterior. Para algunos, América Latina ofrecía oportunidades ilimitadas. En 1929, G. Butler Sherwell, ejecutivo de Baker, Kellog \& Company y antiguo funcionario del Departamento de Comercio de los Estados Unidos y asesor de Hoover para asuntos latinoamericanos, reproduciendo quizás el optimismo de su presidente, afirmaba: "The strikingly rapid economic development of South America is one of these phenomena which the present generation has had the privilege of witnessing... An economic giant is awakening with the aid of American capital" 18 .

Como se sabe, la llegada de Franklin Delano Roosevelt a la presidencia de los Estados Unidos trajo una nueva concepción de las relaciones hemisféricas que quedó resumida en la fórmula de la "política de la buena vecindad". Aunque en cierta manera, esta nueva política había sido iniciada por Hoover, FDR la profundizó y la dirigió hacia campos no explorados hasta entonces. Esta política quedó enmarcada en una reconceptualización de la idea del gobierno mundial llevada a cabo por las elites liberales internacionalistas estadounidenses en las que se intentaba reconstruir el multilateralismo como procedimiento de resolución de los conflictos y sobre todo como garantía de prosperidad para las sociedades capitalistas, es decir, en última instancia, una base para la estabilidad futura. Este proceso estuvo acompañado de un intento de fortalecimiento de la Sociedad de Naciones por parte de esas mismas eli-

15 Costigliola, 1979, p. 101

16 Costigliola, 1979, p. 93.

17 Ver Barbero y Regalsky, 2003, en particular el trabajo de Rocchi, 2003; König - RinKe, 2004; y Pérez, 1999. El proceso de "americanización" en América Latina y Europa fueron obviamente diferentes, pero igualmente complejos. Para el caso europeo ver De Grazia, 2005.

18 G. Butler Sherwell, "The Economic Awakening of South America", Archivo del Banco Central de la República Argentina, Bibioteca Tornquist: Economía 1834-5174, 20 Junio 1929), p.1. 
tes. Por su parte, la SN, como se señaló, había comenzado a mirar más allá de Europa y a dar importancia a regiones como China o América Latina. El estallido de la guerra en 1939 acentuaría estas tendencias.

Hubo una creciente convergencia entre el "Panamericanismo" y el multilateralismo internacional propuesto por la Liga. Algunos ejemplos lo muestran. En el caso de la Organización de Salud de la SN, el interés por América Latina creció desde mediados de la década de 1920, en un momento en que varios países de la región amenazaban con dejar la Liga. La Organización de Salud encargó entonces varias misiones dirigidas hacia América Latina con el objetivo de mostrar a los países americanos que la institución ginebrina se ocupaba de sus intereses. Lo interesante es que la realización de esas misiones no hubiera sido posible sin el apoyo financiero de la Fundación Rockefeller y de la propia actividad del International Health Department de la Fundación en América Latina que tenían una tradición anterior a la de la propia SN. El IHD fue también fundamental en la realización de intercambios de médicos que la SN quería desarrollar. Estas actividades se intensificaron a partir de los años treinta. Lo interesante es que las tres patas (Fundación Rockefeller, Organización de Salud de la Sociedad de Naciones y gobiernos latinoamericanos) que construían esta historia tenían objetivos diferentes. La primera buscaba aplicar medidas de "erradicación" de las epidemias, en lo que constituía una política de objetivos supuestamente limitados y alcanzables. La segunda había desarrollado un concepto más amplio, de salud preventiva, en el que las políticas económicas y sociales debían acompañar a las sanitarias. Los terceros buscaban en las misiones externas apoyos que les permitieran construir sistemas de salud pública nacionales, que representaban también un avance de la centralización estatal ${ }^{19}$. En los años treinta y cuarenta, aunque fuera de manera conflictiva, estos tres objetivos parecieron converger.

Una evolución similar puede encontrarse en el terreno de la economía. Aquí desempeñó un papel significativo el Secretario de Estado norteamericano desde 1933, Cordell Hull. Hull intentó reforzar el Comité Económico de la SN y convencer a los gobiernos latinoamericanos de su importancia. Al mismo tiempo, Hull fue clave en transformar la política comercial estadounidense hacia América Latina de un estricto bilateralismo hacia el multilateralismo ${ }^{20}$. Esta política formaba parte de un resurgir del librecambismo estadounidense, tras un período fuertemente proteccionista en la segunda mitad de los años veinte. En 1934, el presidente Roosevelt aprobó la Reciprocal Trade Agreement que ofrecía bajadas de tarifas a través de acuerdos bilaterales. La mayoría de los firmantes de los acuerdos fueron países latinoamericanos ${ }^{21}$. Roosevelt recuperaba así la idea, presente en Wilson y también en los imperialistas estadounidenses del siglo XIX, de que el libre comercio internacional era un pilar ineludible de la civilización y la paz internacional. Algo similar ocurrió en el campo impositivo, en el que el gobierno Roosevelt rompió con la postura aislacionista anterior y comenzó a apoyar la idea de la cooperación internacional sobre todo con el objetivo de evitar la evasión a través de las fronteras ${ }^{22}$.

\footnotetext{
19 Sobre estas cuestiones ver los ensayos contenidos en CuETo, 1994 y Dubin, 1995.

20 Clavin, 2013, p. 238 y Abreu, 1988, p. 177.

21 Nolan, 2012, p. 116.

22 FARquet, 2014, pp. 460-461.
} 
La convergencia de Panamericanismo y rescate de la Sociedad de Naciones por parte de los liberales internacionalistas estadounidenses no resulta tan contradictorio como pudiera parecer a simple vista y en realidad forma parte de los proyectos de concreción de las ideas wilsonianas que estos liberales estaban llevando a cabo en las décadas de 1930 y 1940. El propio presidente Wilson había propuesto en 1917 como solución al problema de la paz mundial que "the nations should with one accord adopt the doctrine of President Monroe as the doctrine of the world" y de hecho la Doctrina Monroe fue incluida en el Pacto de la Liga ${ }^{23}$. Esta convergencia entre regionalismo y globalismo se observa con bastante claridad en el caso de la interacción de los Estados Unidos con el Comité Fiscal de la SN.

\section{LA CUESTIÓN FISCAL}

Las acciones de Estados Unidos encaminadas a integrar a América Latina en un marco multilateral se multiplicaron en los años 30 . Un buen ejemplo de ello lo proporcionan las relaciones que se establecieron entre los representantes de estadounidenses y el Comité Fiscal de la SN. El Comité Fiscal se ocupaba de la cuestión de la doble imposición y la evasión fiscal internacional, una de las cuestiones más claramente enraizadas en la percepción de un mundo interdependiente y que por lo tanto necesitaba de mecanismos reguladores internacionales ${ }^{24}$.

Las vinculaciones de algunos estadounidenses con el Comité Fiscal se remontaban a su propio origen. El Comité Fiscal permanente de la SN había sido creado como resultado de la Conferencia Fiscal celebrada en 1928 en Ginebra. En esa Conferencia, los delegados norteamericanos, vinculados al Departamento del Tesoro, llevaron la voz cantante. La recuperación de la estabilidad económica en Europa en la segunda mitad de la década de 1920 había requerido la participación activa de los Estados Unidos y a partir de ese momento todas las reuniones económicas internacionales reconocían la necesidad de contar con el compromiso de la potencia americana. Además de los representantes del gobierno, la Fundación Rockefeller, como en el caso de la Organización de Salud, comenzó a subsidiar las actividades del Comité Fiscal. El principal interés de la Fundación residía en encontrar un marco internacional para los gravámenes sobre las multinacionales, tema que se convirtió en el centro de atención del Comité25.

En los primeros años de funcionamiento, el Comité apenas se ocupó de cuestiones no europeas. Pero con la situación en Ginebra cada vez más complicada por las sucesivas crisis en el viejo continente y la creciente importancia de los Estados Unidos

23 Citado en Mazower, 2012, p. 124.

24 En 1943, Mendardo Goytia, delegado chileno en el Comité Fiscal de la Liga observaba: "La estructura económica del mundo moderno ha alcanzado un grado tal de complejidad y ha entrelazado los intereses de los diversos países de un modo tran (sic) estrecho que aun, en el orden fiscal, los sistemas tributarios, creados por los distintos Estados para satisfacer las necesidades de su Erario y cuya esfera de acción debería circunscribirse al territorio nacional, proyectan, sin embargo, su influencia más allá de esas fronteras, para afectar a la economía de países ajenos al establecimiento de aquellos sistemas . . . Entre las consecuencias más inmediatas de esta interdependencia económica está el fenómeno de la doble tributación”. GoYTiA, 1943.

25 F/Fiscal/Session/PV, Minutes of the Fiscal Committee of the LON, 1929-1931. 
que terminaría con el traslado de las secciones técnicas de la Sociedad a Princeton, los países latinoamericanos cobrarían relevancia.

En 1937, el Comité Fiscal había invitado a varias naciones latinoamericanas a participar en lo que constituiría una primera conferencia regional sobre cuestiones fiscales que se celebraría en Ciudad de México en 1940. Uno de los países invitados fue Venezuela, quien rechazó la invitación argumentando que los temas que debían debatirse en esa conferencia no eran de su interés. En 1938 Venezuela debía negociar la renovación de un importante acuerdo comercial con los Estados Unidos. Las negociaciones se extendieron hasta 1939. El gobierno venezolano esperaba obtener de las autoridades estadounidenses una política más benevolente hacia sus exportaciones. El gobierno de los Estados Unidos pudo utilizar estas negociaciones como un arma de presión para obtener otros resultados. También, como parte de esa negociación y estimulado por la necesidad de acometer varias reformas económicas, el gobierno de Venezuela solicitó a los Estados Unidos que enviaran una misión al país, la llamada Misión Fox ${ }^{26}$. No está claro si esta misión y las negociaciones comerciales pudieron haber influido en la posición venezolana con respecto a la conferencia fiscal de la $\mathrm{SN}$, pero es obvio que el gobierno de Estados Unidos contaba aquí con un poderoso instrumento de persuasión. Como las misiones Kemmerer, la Misión Fox recomendó importantes reformas fiscales y el gobierno venezolano creó un Comité de Reforma Impositiva con el objetivo de estudiar y aplicar las medidas propuestas por la misión. El secretario del Comité Fiscal de la Liga, el belga Paul Deperon, cuando visitó Venezuela, se entrevistó con este Comité de Reforma. Venezuela finalmente participó en la conferencia de México de 1940 y según Deperon en su reforma impositiva estaba guiándose por los principios discutidos en esa conferencia ${ }^{27}$.

Pero el objetivo de acercar América Latina a los organismos técnicos de la SN no sólo se llevaba a cabo a través de presiones. Los Estados Unidos también propiciaban reformas en sus políticas internas que facilitaban esa estrategia. Por ejemplo, en 1941, el Congreso estadounidense introdujo reformas en las leyes fiscales que buscaban eliminar discriminaciones contra los contribuyentes del hemisferio y aliviar la carga fiscal para las empresas estadounidenses que desarrollaran su actividad en América Latina. Esta medida era crucial, ya que al eximir en buena parte de sus gravámenes a las multinacionales estadounidenses Washington estaba preparando el terreno para la realización de acuerdos fiscales de doble imposición con los países latinoamericanos. Detrás de esta política estaba un abogado especialista en impuestos, con una larga experiencia internacional, Mitchell B. Carroll. Carroll había sido el miembro de Estados Unidos en el Comité Fiscal de la SN desde su creación en 1928 y además tenía una vinculación estrecha con la International (y la American) Chamber of Commerce, y por tanto con aquellos grandes empresarios estadounidenses orientados hacia el mercado mundial. Según Carroll, las reformas fiscales impulsadas por el Congreso no

26 U. S. Advisory Economic Mission to Venezuela, 1940. Para las negociaciones del acuerdo comercial y la petición de Venezuela de un mejor tratamiento a sus exportaciones, ver Frus, United States Department of State, diplomatic papers, 1939. The American Republics (1939), pp. 805-808 and Frus, United States Department of State, diplomatic papers, 1940. The American Republics (1940), pp. 1176-1179.

27 Deperon to Loveday, Río de Janeiro, November 8th, 1941, LON, C 1643 Record Group 8 (Princeton Office). Financial Section and Economic Intelligence Sevice. Fiscal Committee. 
eran sólo un incentivo empresarial, sino que "the amendment of the Internal Revenue Code bespeaks a policy of bringing about hemisphere economic solidarity through the adoption in treaties of principles of international tax law" 28 .

Los internacionalistas de Estados Unidos y los tecnócratas de la SN se necesitaban mutuamente en su estrategia hacia América Latina. Las distintas misiones, conferencias y políticas llevadas a cabo se originaban así en las discusiones entre el secretario de la Sección Económica o el Comité Fiscal de la SN, altos funcionarios del Departamento de Estado de los Estados Unidos, representantes de la Fundación Rockefeller, y empresarios o abogados como Carroll. La posibilidad de que los intereses del gobierno de los Estados Unidos y de los expertos de la SN se percibieran como idénticos era una realidad. Sean Lester, Secretario General de la Liga, lo mencionó en su correspondencia con Alexander Loveday, secretario de la Sección Financiera de la Sociedad. En 1941, cuando se estaba discutiendo la posibilidad de una segunda conferencia americana sobre cuestiones fiscales, Lester le recordó a Loveday que "there are obvious reasons why any meeting, if held, should be kept well within the frame-work of the League Organization, perhaps all the more so since you would be organizing it from U.S. soil". La principal razón para ello era que el "emphasis on the League auspices, might contribute towards the success of your meeting in view of the sometimes delicate inter-American relations and balance of interests" ${ }^{29}$. (Aunque quizás Lester estaba también preocupado por la pérdida de influencia europea en la SN tras el traslado a Princeton) ${ }^{30}$.

Pero la idea de que la influencia estadounidense era percibida como claramente negativa por los latinoamericanos no es del todo adecuada ${ }^{31}$. De hecho, en las cuestiones técnicas que la Liga deseaba plantear la presencia estadounidense era en muchas ocasiones un activo. En 1940, el miembro brasileño del Comité Fiscal aplaudió la intervención de Carroll en la discusión sobre cuestiones de doble imposición ${ }^{32}$. En 1943, en Bolivia, Héctor Ormachea Zalles, que había sido asesor de la Organización Financiera de la Liga, dimitió de su cargo, y según el miembro boliviano del Comité Fiscal, Vicente Mendoza López, comenzó una campaña pública de descrédito de la SN. Para Mendoza López la opinión de Ormachea Zalles no tenía influencia, pero esto es más que improbable. Ormachea era una figura ampliamente reconocida, se había convertido en Rector de la principal universidad del país y sus ideas eran publicadas y discutidas en la prensa ${ }^{33}$.

28 CARroll, 1941, p. 794.

29 Sean Lester to Loveday, March 7, 1941, LON C 1645 Record Group 8 (Princeton Office). Financial Section and Economic Intelligence Service. Fiscal Committee. Registered Files of Correspondence and Memoranda, 1940-1946.

30 Clavin, 2013, p. 273.

31 Muchos historiadores han señalado la extensión del sentimiento anti-imperialista (es decir, anti-yankee) en América Latina en el período de entreguerras. Sin embargo, este relato no toma en cuenta la complejidad de la región ni la popularidad de FDR en muchas repúblicas latinoamericanas. Para el caso argentino, véase las originales contribuciones de RUIZ JIMÉNEZ, 1998 y s/a.

32 Joao Lyra, Mexico, June 13rd, 1940, LON, C 1644 Record Group 8 (Princeton Office). Financial Section and Economic Intelligence Service. Registered Filed of Correspondence and Memoranda, 1910-1946.

33 Vicente Mendoza López to Deperon, La Paz, August 23rd, 1943, LON C 1645 Record Group 8 (Princeton Office). Financial Section and Economic Intelligence Service. Fiscal Committee. Registered files of Correspondence and memoranda, 1940-1946. 
El ejemplo más claro de desconfianza hacia la Liga afectó a la organización de la segunda conferencia regional del Comité Fiscal. En principio, los burócratas de la SN esperaban que Colombia organizara la conferencia y varios representantes del gobierno de ese país habían expresado su deseo de hacerlo. Sin embargo, en 1942, Francisco Urrutia, el miembro colombiano del Comité Fiscal, informó a Deperon de que la conferencia tenía que posponerse. La clave de la decisión, según la información confidencial de Urrutia, fue la oposición personal del presidente colombiano, Alonso López Pumarejo:

As you know Dr. Alfonso López was the author of the project for an American League of Nations, which was discussed in the last Pan American Conference. Although he did not present this project as an attack to the Geneva organisation, he justified his project with a strong criticism of the Geneva organisation (...).

At present Dr. López thinks that it is not a question of retiring from the League of Nations, because it is a duty of all these countries to wait until the war is over... but in various conferences he gave in the United States he said frankly that he wishes that his project of an American League of Nations be considered after the war, showing that he does not have much faith in a universal organization...

We, who have always been convinced of the ideals of the League of Nations, will continue fighting for it with the firm hope that some day we will be able to convince mandataries as pessimistic as Dr. López" ${ }^{34}$.

Finalmente la idea de una conferencia en Bogotá fue descartada y la segunda reunión regional del Comité Fiscal se celebraría en México otra vez. En suma, la asociación con la Liga no era atractiva para muchos latinoamericanos. Una perspectiva regional integrada en el marco del panamericanismo era muchas veces más atrayente que el lenguaje universal de la SN. Para algunos gobiernos y expertos latinoamericanos, la participación de Estados Unidos era una garantía. Desde 1938, en las conferencias Inter-Americanas, los delegados de Estados Unidos recurrieron al lenguaje del "multilateralismo" como una clave para construir sus relaciones con las repúblicas del sur. Para los gobiernos latinoamericanos multilateralismo significaba cooperación regional entre iguales, "that decisions regarding the hemisphere would be taken together, after consultation" ${ }^{35}$. Esto no era necesariamente así para los Estados Unidos. Pero la confianza en la solidaridad de los Estados Unidos sobrevivió hasta por lo menos la creación de la ONU y en algunos casos incluso más allá.

¿Qué esperaban los gobiernos latinoamericanos de su colaboración con Estados Unidos en los comités de la SN? ¿Hasta qué punto influyeron los latinoamericanos en la construcción de la agenda multilateral y de reconstrucción de los sistemas de gobernanza global? La organización y los contenidos de la segunda conferencia del Comité Fiscal en México DF en 1943 es una buena ventana para asomarnos a esta cuestión. Cuando la SN comenzó a sondear a los miembros latinoamericanos del Comité Fiscal estos señalaron que sus gobiernos estaban dispuestos a cooperar en la

34 Francisco Urrutia to Deperon, Bogotá, November 24th, 1942, LON C 1645 Record Group 8 (Princeton Office). Financial Section and Economic Intelligence Service. Fiscal Committee. Registered Files of Correspondence and Memoranda, 1940-1946.

35 Wood, 1985, pp. x-xi. 
celebración de una segunda conferencia si se cumplían varias condiciones. En primer lugar, se pedía una mayor representación latinoamericana. En segundo se reclamaba la inclusión de nuevos puntos en la agenda de discusión que fueran más allá de las cuestiones sobre doble imposición. Los gobiernos latinoamericanos esperaban que la conferencia se ocupara también de asuntos de cooperación entre las administraciones fiscales y de la relación entre las autoridades nacionales y locales en un sistema. Dicho de otra manera, los gobiernos latinoamericanos esperaban contar con una transferencia del conocimiento técnico de la SN para aplicarlo a sus reformas fiscales nacionales, que entre otros aspectos implicaban una centralización administrativa.

Muchas de las peticiones de los latinoamericanos fueron incorporadas a la agenda de la conferencia. Esto tenía que ver de nuevo con un proceso de convergencia en los intereses. Desde finales de 1942 y principios de 1943, los aliados empezaron a ver con más optimismo las posibilidades de victoria en la guerra contra el Eje y la idea de pensar en la reorganización del orden económico mundial ganó cada vez más peso. La conferencia mexicana sobre cuestiones impositivas fue vista así "as part of the postwar program"36. Los problemas internos sobre cuestiones fiscales se incluyeron en la discusión de la conferencia porque los países latinoamericanos habían solicitado un estudio de los principios técnicos sobre materia impositiva, pero también porque "it was desirable to have a general discussion about common internal tax problems, in order to elicit directives for studies on postwar fiscal problems" ${ }^{37}$.

Uno de los aspectos cruciales de la discusión en la Conferencia de 1943 fue el de los posibles acuerdos sobre doble imposición con respecto a los beneficios del capital (móvil) y de las empresas multinacionales. En 1942, el miembro argentino del CF, Ernesto Malaccorto, envió una carta al resto de representantes sudamericanos en el Comité con el objetivo de crear un frente unido para discutir con los burócratas de la SN y los representantes de Estados Unidos. Su propuesta fue ampliamente discutida por los expertos latinoamericanos y generó un debate intenso entre los técnicos de la SN. Las propuestas de Malaccorto se inscribían en una línea que podríamos denominar "desarrollista": "these countries imperiously need to develop their foreign trade and... obtain the help of foreign capital in order to encourage their industries if they aspire at all to a similar development to that of the great nations" 38 . La crisis de 1929 y el estallido de la Segunda Guerra Mundial habían significado un impulso para las manufacturas de los países grandes y medianos latinoamericanos y en muchos de

\footnotetext{
36 Mitchell Carroll, "A Clipping on the First Conference of the Commission of Inter-American Development”, May 11, 1944, LON C 1645 Record Group 8 (Princeton Office). Financial Section and Economic Intelligence Service. Fiscal Committee. Registered Files of Correspondence and Memoranda, 1940-1946.

37 League of Nations. Fiscal Committee. Second Regional Conference in Mexico, July 19-29, 1943. Account of the Proceedings by the Secretary, LON C 1645 Record Group 8 (Princeton Office). Financial Section and Economic Intelligence Service. Fiscal Committee. Registered Files of Correspondence and Memoranda, 1940-1946.

38 League of Nations. Fiscal Committee. Second Mexico Regional Tax Conference. Prevention of Double Taxation through Income and Property Taxes. Note dated February 1942 by Dr. Ernesto Malaccorto, Argentine corresponding member of the Fiscal Committee y "Translation of a Letter and Memorandum Sent by Dr. Ernesto Malaccorto, Director General of Income Tax, Argentina, to the South American Members and Corresponding Members of the Fiscal Committee of the League of Nations," ambos en LON C 1646. Record Group 8 (Princeton Office). Financial Section and Economic Intelligence Service. Fiscal Committee. Registered files of Correspondence and Memoranda, 1940-1946. (30) 72.
} 
ellos se pensaba que una economía sana tras el final del conflicto no podría significar el retorno a una mera posición de exportadores de materias primas. Malaccorto (y otros muchos latinoamericanos) aspiraban a atraer el capital extranjero y al mismo tiempo poner en marcha políticas impositivas autónomas que permitieran a las repúblicas latinoamericanas profundizar en su desarrollo industrial. La perspectiva de Malaccorto era fuertemente regional y cuando se hablaba de atraer el capital foráneo se estaba pensando principalmente en los Estados Unidos. Esto simplificaba los problemas: "the continental problem presents peculiar features as there is only one great capital exporting country and a large number of capital importing countries which, if they would agree on a determinate stand, might perhaps obtain the capital exporting country the solution they desire" 39 .

La visión de Malaccorto era la de una interdependencia regional en la que la acción colectiva de los países latinoamericanos debería servir para moldear las respuestas de Estados Unidos, en cierta medida anticipando la que será la postura de la CEPAL en la segunda posguerra. ¿Con qué objetivos? En la propuesta de Malaccorto, el fin era preservar para los Estados latinoamericanos el derecho a gravar los beneficios del capital mobiliario en un supuesto acuerdo internacional sobre doble imposición. Es decir, había que persuadir a Estados Unidos para que renunciara a la recaudación de impuestos sobre los beneficios realizados por sus ciudadanos en inversiones en el exterior. Este plan era factible actuando de manera conjunta y, además, según Malaccorto, contaba con el apoyo de la SN: "we think -and Mr. Deperon shares our views- that if South American countries should present in an international conference a unanimous opinion... they could obtain an acceptance of principle from the United States" ${ }^{40}$. Sin embargo, el apoyo de Deperon no está claro. En una copia de la nota de Malaccorto archivada junto a los papeles del secretario del Comité Fiscal se encuentra escrito en el margen un signo de exclamación y otro de interrogación. No podemos saber si era un comentario del propio Deperon. En todo caso, en abril de 1942 Deperon escribió a Carroll señalando: "I have the impression that Dr. Malaccorto's memorandum deserves a close study, specially as his views concerning some points where he takes exception to the provision of the draft (1940), are perhaps less divergent from it than it may at first sight appear... I should appreciate it if you would regard this communication as confidential" ${ }^{41}$. Más allá de la influencia que pudiera tener la intervención de Malaccorto, está claro que el Comité Fiscal quería asegurarse un resultado exitoso de la Segunda Conferencia Regional y entendía que para ello deberían aceptarse tanto como fuera posible las peticiones de los representantes latinoamericanos.

Incluso sin un frente latinoamericano unido, la posición de Malaccorto tenía oportunidades de triunfar. Él mismo señalaba las ventajas que los Estados Unidos podían obtener de este "sacrificio fiscal". Esta medida estimularía las inversiones estadounidenses en el exterior al eliminar la doble imposición (ahora esas inversiones sólo

\footnotetext{
39 Ibídem.

40 Ibídem.

41 Deperon to Carroll, April 20, 1942, LON C 1646. Record Group 8 (Princeton Office). Financial Section and Economic Intelligence Service. Fiscal Committee. Registered files of Correspondence and Memoranda, 1940-1946. (30) 72.
} 
pagarían en el país receptor) y también porque las alícuotas latinoamericanas eras más bajas que las estadounidenses.

De hecho, las políticas impositivas estadounidenses se habían movido en la dirección que facilitaba el acuerdo. En 1942, el Congreso de los Estados Unidos había aprobado una ley que eximía a las sociedades de la sobretasa en el impuesto a la renta para las corporaciones si estas empresas habían llevado a cabo sus negocios en el hemisferio occidental y obtenían $95 \%$ de sus ingresos brutos de fuentes externas a los Estados Unidos ${ }^{42}$. Esta medida intentaba estimular el comercio hemisférico pero también facilitaba la posibilidad de alcanzar acuerdos de doble imposición con los países de la región, ya que les permitía gravar a estas empresas en la fuente donde obtenían sus beneficios. En un momento en que la Segunda Guerra Mundial permitió a Roosevelt incrementar las tasas del impuesto personal a la renta hasta el $90 \%$ y cuando las corporaciones se vieron obligadas a pagar un mayor impuesto a la renta en Estados Unidos, las empresas multinacionales obtuvieron un tratamiento extremadamente beneficioso ${ }^{43}$. Esto facilitó los acuerdos que se alcanzaron en la conferencia de 1943. Allí, como señaló el representante chileno se había aceptado "el punto de vista de los países menos desarrollados" y los estados receptores de capital habían conservado el derecho a gravar los beneficios de las multinacionales ${ }^{44}$.

Igualmente importante fue la cuestión de la explotación de las materias primas y minerales. Tradicionalmente, los países exportadores de capital habían mantenido que el beneficio derivado de las actividades extractivas (como la minería) se producía en el momento de la venta del producto. En 1943, todavía mantenían esa postura. Los latinoamericanos por supuesto pensaban de manera diferente. Mendardo Goytia, miembro chileno del Comité Fiscal, indicaba que la Conferencia de 1943 "felizmente" había aceptado el principio de que los países productores tenían derecho a gravar los beneficios que se obtenían de la explotación de minas y de otras materias primas.

Uno de los aspectos más interesantes de la discusión sobre las formas de organización económica global fue la creación dentro de la Conferencia fiscal de 1943 del llamado Sub-Comité IV, encargado de tratar con problemas fiscales generales. Esta había sido una reclamación de los países latinoamericanos, en particular México, y su composición reflejaba intereses que iban más allá de las cuestiones impositivas. Su presidente era Jesús Echeverri Duque, un abogado y político colombiano y sus otros miembros eran Jesús Sáenz, Director General de Estadísticas de México, Raúl Martínez Ostos, experto del Banco de México, y Mario La Gamma, asesor del Ministerio de Hacienda de Uruguay (y quien sería uno de los expertos latinoamericanos en la Conferencia de Bretton Woods). Las propuestas del Sub-Comité IV fueron radicales, uniendo las cuestiones de impuestos en el mundo de posguerra a la profundización de ambiciosas políticas económicas y sociales: "taxation was to be geared as to im-

42 Mitchell Carroll, "A Clipping on the First Conference of the Commission of Inter-American Development", May 11, 1944, LON C 1645 Record Group 8 (Princeton Office). Financial Section and Economic Intelligence Service. Fiscal Committee. Registered Files of Correspondence and Memoranda, 1940-1946.

43 WebBer - Wildavsky, 1986 p. 485 y Jones, 1996. Una interesante comparación entre el tratamiento de las empresas multinacionales y las inversiones en el extranjero en Estados Unidos y Gran Bretaña se encuentra en Piccioto, 1992, p. 18.

44 Goytia, 1943. 
prove the distribution of wealth, and at the same time, not inhibit individual initiative... tax problems of the day could not be dissociated from a consideration from long social and economic objectives" ${ }^{\prime 5}$. La propuesta encontró una recepción fría entre algunos representantes conservadores de países latinoamericanos que consideraban "highly objectionable the partiality it showed to cyclical budgeting, capital budgets, progressive taxation". Para evitar mayores conflictos, el Comité Fiscal decidió no adoptar ninguna resolución sobre esta declaración y el borrador del Sub-Comité IV fue enviado a Princeton para un estudio más detenido ${ }^{46}$.

Al parecer, los expertos de Estados Unidos no se opusieron a la propuesta del Sub-Comité IV. Deperon afirmó que estas ideas "reproduced many ideas of the "Transition' report". Con ello hacía referencia al documento The Transition from War to Peace Economy, publicado por la Liga, y en particular a la idea de que "tax systems had to be so organized and managed that fiscal policy should always conform in its effects to general economic and financial policy" 47 . Como ha subrayado Patricia Clavin, el Transition Report, una de las más exitosas publicaciones de la SN, adoptó una perspectiva global, que tenía como objetivo mejorar el bienestar de la humanidad. Era, afirma Clavin, una especie de proto-teoría de la modernización ${ }^{48}$. Como mencionaba Deperon, los expertos latinoamericanos conocían los borradores del Transition Report.

La idea de discutir cuestiones impositivas no sólo con el objetivo de estimular el comercio internacional sino también desde el punto de vista de las necesidades internas (desarrollo industrial en muchos casos) de los países latinoamericanos era uno de los aspectos que resultaba más atractivos para los latinoamericanos. Hay que subrayar, empero, que el "desarrollismo" casaba con la postura liberalizadora de los Estados Unidos. Como se vio, permitir a los países latinoamericanos gravar las empresas multinacionales norteamericanas fue en el fondo una medida de alivio fiscal para éstas. Además, las conferencias de 1940 y 1943 consagraron el principio de "arm's lenght principle", que el propio Carroll había desarrollado primero en la Cámara de Comercio de los Estados Unidos y posteriormente en su participación en el Comité Fiscal de la SN. Ese principio significaba que las empresas multinacionales no podían ser tratadas a efectos impositivos de manera diferente que las empresas nacionales. Aunque las consecuencias de esta política necesitan ser exploradas con detenimiento, podría implicar que estas empresas se beneficiaban de no ser consideradas como una sola unidad y no recibir un gravamen sobre sus utilidades globales y que podían recurrir a mecanismos de tergiversación de sus cuentas a través de la manipulación de los precios de transferencia de insumos entre las distintas subsidiaras (el llamado "transfer pricing") ${ }^{49}$.

¿Cómo explicar la apuesta por el multilateralismo y la Sociedad de Naciones como mecanismo de intervención en América Latina? Ya se ha subrayado cómo las medidas propuestas contribuían a estimular el libre cambio y las actividades de las empresas

\footnotetext{
45 League of Nations, Second Regional Conference, 1943.

46 Ibídem.

47 Ibídem y League of Nations, 1943, parte I.

48 Clavin, 2013, pp. 285 y ss.

49 Markham, 2006, pp. 14-18.
} 
estadounidenses. Además, la mezcla de liberalismo internacional e intervencionismo dentro de las fronteras se había convertido en una especie de leit-motiv ideológico para la administración Roosevelt e influía fuertemente su política de buena vecindad. Constituía lo que politólogos e historiadores han denominado posteriormente "embedded liberalism" ("liberalismo regulado"). Pero aún así, estos objetivos se podrían haber perseguido por fuera de las estructuras de la Sociedad de Naciones, ya fuera de manera bilateral o limitándose al territorio del panamericanismo. Los tres mecanismos se utilizaron, como se ha visto en el caso de las políticas fiscales, a veces al mismo tiempo. Aquí sólo se puede aventurar algunas hipótesis sobre el recurso a la Sociedad de Naciones. Obviamente, los liberales internacionalistas estadounidenses y los expertos de la SN coincidieron en muchas de sus posiciones. En segundo lugar, podría pensarse que desde el principio la idea no era sólo acercarse a América Latina sino reconstruir el orden internacional mundial, tomando América Latina como base. Por último, las décadas de 1930 y 1940 significaron cambios dramáticos para la sociedad norteamericana, que sufrió la Gran Depresión y se vio sumergida a partir de 1941 en un nuevo conflicto mundial. Al mismo tiempo, la administración Roosevelt introdujo modificaciones profundas en la relación entre Estado y sociedad civil, de lo que da buena prueba la profundización de la capacidad recaudatoria ya mencionada. Cuando las sociedades se ven sometidas a tensiones domésticas profundas y a vinculaciones externas muy complejas, las elites políticas buscan plantear los debates en una escala mayor, recurriendo a un conocimiento externo y supuestamente técnico y neutral, el producido por las llamadas "epistemic communities" ${ }^{50}$. Es quizás aquí donde convergieron las nuevas formas de intervencionismo en América Latina y el recurso a la cooperación con los organismos técnicos de la Sociedad de Naciones.

Aunque hemos hablado en este artículo de una forma de intervencionismo, sería un error pensar que los latinoamericanos desempeñaron un papel pasivo en la construcción de este modelo de pensamiento. De hecho, como se ha visto, el Sub-Comité IV de la Conferencia de 1943 había sido una creación latinoamericana que respondía a las necesidades de los gobiernos de la región ${ }^{51}$.

\section{BALANCE: LOS LÍMITES DEL PANAMERICANISMO}

Como se dijo, los latinoamericanos vivieron la Conferencia de 1943 como un éxito. Este se logró con la cooperación entre los países de la región pero también gracias al "amplio espíritu de equidad y de comprensión de las necesidades de nuestros

50 Un análisis clásico sobre esta cuestión en HAAs, 1990, capítulo 7.

51 De hecho, además de los países representados en el Sub-Comité IV, varias personalidades políticas de la región, como el presidente y el Ministro de Exteriores chilenos, habían participado en la redacción de un borrador previo. Ver Vicente Mendoza López to Deperon, La Paz, August 23, 1943, LON C 1645 Record Group 8 (Princeton Office). Financial Section and Economic Intelligence Service. Fiscal Committee. Registered Files of Correspondence and Memoranda, 1940-1946). Con respecto al concepto de "embedded liberalism" ver RUGGIE, 1985. Los acuerdos de Bretton Woods parecen representar la culminación de esta visión del mundo. Tradicionalmente han sido analizados como parte de la entente anglo-americana para reestructurar la economía internacional. Una contribución reciente e importante STEIL, 2013 continúa esta tradición. La influencia de los latinoamericanos en la construcción del "embedded liberalism” ha sido rescatada por HeLLEINER, 2003 y 2006. 
países manifestada por los Delegados y funcionarios de los países exportadores de capital" 52 . Obviamente, en el contexto de 1943, se estaba haciendo referencia a los estadounidenses que participaron en la Conferencia.

Así pues, los representantes de Estados Unidos ofrecieron condiciones favorables a las repúblicas latinoamericanas. Estados Unidos era un país en guerra y necesita el apoyo de sus vecinos del sur. Sin embargo, el proceso de aceptación de las posiciones latinoamericanas fue gradual y había comenzado en la década de 1930.

En segundo lugar, las peculiaridades del sistema impositivo estadounidense y su deseo de expandir las actividades de sus empresas en el hemisferio occidental desempeñó un papel importante en esta aproximación a la cuestión de la organización económica internacional. En tercer lugar, la dimensión ideológica, lo que se ha denominado "embedded liberalism".

Pero esta dimensión ideológica tenía matices complicados para los latinoamericanos. Los liberales internacionalistas estadounidenses diseñaron aproximaciones multilaterales a América Latina a través de su interacción con la Sociedad de Naciones. Se estaba construyendo aquí un nuevo modelo de imperio, en el que Estados Unidos actuaría como coordinador del capitalismo internacional. En ese modelo América Latina tenía que desempeñar el papel de laboratorio para luego poder exportarlo a Europa al final del conflicto. Mitchell Carroll, quien había importando su experiencia europea en el Comité Fiscal a América Latina, vinculó claramente el establecimiento de lazos económicos más sólidos en el continente americano con una dimensión global, "with the growing confidence in a United Nations victory in Europe and Asia"53.

La defensa del multilateralismo por parte del gobierno de los Estados Unidos y de una parte importante de sus elites había dejado a los latinoamericanos un espacio importante para avanzar sus agendas. No está claro cuando se cerró esta ventana de oportunidad. Incluso después de Bretton Woods, en la conferencia Panamericana de Chapultepec (México) en 1945, la industrialización de América Latina había recibido el beneplácito (matizado) de los Estados Unidos ${ }^{54}$.

Sin embargo, el papel de la Fundación Rockefeller o de personajes como Carroll en Europa y América Latina mostraba una visión más global que regional y una visión en la que Europa seguía siendo la clave del arco de la gobernanza global. En este sentido, las expectativas latinoamericanas sobre la cooperación interamericana pudieran haber sido excesivas.

En 1944, Carroll envió a Deperon un informe sobre los problemas impositivos para el mundo de posguerra en el que mostraba su preocupación por la recuperación europea. El Viejo Mundo iba a necesitar abundantes productos, bienes primarios en una situación de caída de la capacidad adquisitiva. Los impuestos tenían que facilitar

52 Goytia, 1943.

53 Mitchell Carroll, "A Clipping on the First Conference of the Commission of Inter-American Development”, May 11, 1944, LON C 1645 Record Group 8 (Princeton Office). Financial Section and Economic Intelligence Service. Fiscal Committee. Registered Files of Correspondence and Memoranda, 1940-1946.

54 Love, 1996, p. 216. Ver también, Wood, 1985, p. 61; Ross, 2012 y las reflexiones originales de MARICHAL, 2002. 
una caída en los precios de las importaciones que necesitaban los europeos ${ }^{55}$. Esto ponía en duda las concesiones realizadas a América Latina en 1943. Ya en 1942, el miembro ecuatoriano del Comité Fiscal, Eduardo Riofrío, expresaba su temor de que el final de la guerra significase un cambio de política y que los países europeos no aceptasen las propuestas impositivas que habían acordado entre los latinoamericanos, como efectivamente ocurriría en $1946^{56}$. Desafortunadamente para América Latina (y afortunadamente para Europa) la teoría y práctica del "embedded liberalism" que los Estados Unidos habían ensayado en América Latina estaba destinado a servir como modelo para la reconstrucción europea.

\section{REFERENCIA BIBLIOGRÁFICAS}

Abreu, Marcelo de Paiva

1988 "La Argentina y Brasil en los años treinta. Efectos de la política económica internacional británica y estadounidense". En THORP (comp.), América Latina en los años treinta. El papel de la periferia en la crisis mundial. México. FCE.

Barbero, María Inés - Regalsky, Andrés (eds.)

2003 Americanización: los Estados Unidos y América Latina en el siglo XX: transferencias económicas, tecnológicas y culturales. Buenos Aires. Universidad Tres de Febrero.

Bértola, Luis - OcAmpo, José Antonio

2013 Desarrollo, vaivenes y desigualdad: una historia económica de América Latina desde la independencia. Madrid. Secretaría General Iberoamericana.

Bulmer-Thomas, Victor

1994 The Economic History of Latin America since Independence. Cambridge. Cambridge University Press.

CARroll, Mitchell B.

1941 "Development of International Tax Law in the Americas," Law \& Contemporary Problems. Durham, North Carolina, $\mathrm{n}^{\mathrm{o}}$ 8, pp. 793-801.

Clavin, Patricia

2013 Securing the World Economy. The Reinvention of the League of Nations, 1920-1946. Oxford. Oxford University Press.

\footnotetext{
55 Carroll to Deperon, NY, June 15th, 1944. "A general outline of problems of double taxation to be considered in connection with postwar reconstruction prepared for the Nation Association of Manufacturers," LON C. 1646. Record Group 8 (Princeton Office). Financial Section and Economic Intelligence Service. Fiscal Committee. Registered files of Correspondence and Memoranda, 1940-1946. (30) 72.)

56 Riofrío a Deperon, Quito, 15th June, 1942, LON C 1645 Record Group 8 (Princeton Office). Financial Section and Economic Intelligence Service. Fiscal Committee. Registered Files of Correspondence and Memoranda, 1940-1946.
} 
Corol, Juan Carlos

2011 "La economía". En Cattaruzza, Crisis económica, avance del estado e incertidumbre política (1930-1943). Tomo VII de la Nueva Historia Argentina. Buenos Aires. Sudamericana, pp. 17-47.

Costigliola, Frank

1979 'American Foreign Policy in the 'Nut Cracker'. The United States and Poland in the 1920s". The Pacific Historical Review. Berkeley, no 48/1, pp. 85105.

Cueto, Marcos (ed.)

1994 Missionaries of Science. The Rockefeller Foundation \& Latin America. Bloomington. Indiana University Press.

De Grazia, Victoria

2005 Irresistible Empire: America's Advance Through Twentieth-Century Europe. Cambridge. Belknap Press.

DRAKE, Paul

1989 The Money Doctor in the Andes: The Kemmerer Missions, 1923-1933. Durham. Duke University Press.

DuBIN, Martin David

1995 "The League of Nations Health Organisation". En WeIndLING (ed.), International Health Organisations and Movements, 1918-1939. Cambridge. Cambridge University Press.

FARQUET, Christophe

2014 La défense du paradis fiscal suisse avant la Seconde Guerre mondiale: una histoire internationale. Analyse de la politique helvétique au sein des négociations sur la doublé imposition et l'évasion discale durant l'entre-deuxguerres. Lausanne. Université de Lausanne (Tesis Doctoral).

Ferguson, Niall - Maier, Charles S. - Manela, Erez - Sargent, Daniel J. (eds.)

2010 The Shock of the Global. The 1970's in Perspective. Cambridge. Harvard University Press.

FisCHER, Thomas

2012 Die Souveränitat der Schawachen. Lateinamerika und der Völkerbund, 19201936. Stuttgart. Franz Steiner Verlag.

Frus United States Department of State - Foreign Relations of the United States Diplomatic PAPERS

1939 The American Republics. Volume V. U.S. Government Printing Office, http:// digital.library.wisc.edu/1711.dl/FRUS.FRUS1939v05, consultado 20/12/2014 .

1940 The American Republics. Volume V. U.S. Government Printing Office, http:// digital.library.wisc.edu/1711.dl/FRUS.FRUS1940v05, consultado 20/12/2014.

Goytia, Mendardo

1943 "La Conferencia Tributaria de México. Problemas sobre doble tributación", Revista de Economía y Finanzas. Santiago de Chile, septiembre, s/n, s/pp. 
Grandin, Greg - JosePH, Gilbert M. (eds.)

2010 A Century of Revolution: Insurgent and Counterinsurgent Violence during Latin America's Long Cold War. Durham. Duke University Press.

HaAs, Ernst B.

1990 When Knowledge is Power. Three Model of Change in International Organizations. Berkeley. University of California Press.

HELLEINER, Eric

2003 "The Southern Side of 'Embedded Liberalism"”. En Flandreau (ed.), Money Doctors: The Experience of International Financial Advising 1850-2000. London. Routledge.

HELLEINER, Eric

2006 "Reinterpreting Bretton Woods: International Development and the Neglected Origins of Embedded Liberalism". Development and Change. London, $\mathrm{n}^{\circ}$ 37/5, pp. 943-967.

HugGins, Martha

1998 Political Policing: The United States and Latin America. Durham. Duke University Press.

JoNES, Carolyn

1996 "Mass-based Income Taxation". En BrownleE (ed.), Funding the Modern American State, 1941-1995. Cambrige. Cambridge University Press.

Joseph, Gilbert M.

2008 "What We Know Now and Should Know: Bringing Latin America More Meaningfully into Cold War Studies". En JosePH - SPENSER (eds.), In from the Cold. Latin America's New Encounters with the Cold War. Durham. Duke University Press.

KöNIG, Hans-Joachim - RinKE, Stefan

2004 North Americanization of Latin America? Culture, Gender, and Nation in the Americas. Stuttgart. Dieter Heinz.

KoskenNiemi, Marti

2005 El discreto civilizador de naciones. El auge y la caída del derecho internacional 1870-1960. Buenos Aires. Ciudad Argentina.

League of Nations

1939 The Development of International Co-operation and Social Affairs. Ginebra. Sociedad de Naciones.

League of Nations

1943 The Transition from War to Peace Economy: Report of the Delegation on Economic Depressions. Ginebra. Sociedad de Naciones.

Love, Joseph L.

1996 "Economic Ideas and Ideologies in Latin America since 1930". En BetHeLL (ed.), Ideas and Ideologies in Twentieth Century Latin America. Cambridge. Cambridge University Press. 
Marichal, Carlos

2002 "Introducción". En Marichal. (coord.), México y las Conferencias Panamericanas 1889-1938: Antecedentes de la globalización. México. Secretaría de Relaciones Exteriores.

MAZOwER, Mark

2012 Governing the World. The History of an Idea. London. Allan Lane.

MARKHAM, Michelle

2006 The Transfer Pricing of Intangibles. The Hague. Kluwer Law International.

Nolan, Mary

2012 The Transatlantic Century. Europe and America, 1890-2010. Cambridge. Cambridge University Press.

O'Rourke, Kevin H. - Williamson, Jeffrey G.

2006 Globalización e Historia. La evolución de la economía atlántica en el siglo $X I X$. Zaragoza. Prensas Universitarias de Zaragoza.

PÉREZ JR., Louis

1999 On Becoming Cuban: Identity, Nationality, and Culture. Chapell Hill, NC. North Carolina University Press.

PettinÀ, Vanni

2011 Cuba y Estados Unidos, 1933-1959. Del compromiso nacionalista al conflicto. Madrid. Catarata.

Piccioto, Sol

1992 International Business Taxation: A Study in the Internationalization of Business Regulation. Londres. Weidenfeld and Nicholson.

RocchI, Fernando

2003 "Americanización del consumo: las batallas por el mercado argentino, 19201945”. En Barbero - Regalsky (eds.), Americanización: los Estados Unidos y América Latina en el siglo XX: transferencias económicas, tecnológicas y culturales. Buenos Aires. Universidad Tres de Febrero.

ROSENBERG, Emily S.

1987 World War I and the Growth of United States Predominance in Latin America. Nueva York. Garland.

Ross, César

2012 "La Carta Económica de las Américas, 1945: el disenso de Chapultepec". Estudios Latinoamericanos. Valparaíso, no 8, pp. 57-82.

RugGIE, John

1982 "International Regimes, Transaction, and Changes: Embedded Liberalism in the Postwar Economic Order". International Organization. Boston, n ${ }^{\circ} 36 / 2$, pp. 379-405.

RUIZ JIMÉNEZ, Laura

1998 “Peronism and Anti-Imperialism in Argentinian Press: 'Braden or Perón' was also 'Perón is Roosevelt'”. Journal of Latin American Studies. Cambridge, ${ }^{\circ}$ 30/3, pp. 551-572. 
RuIZ JiMÉNEZ, Laura

s.a. "De la desconfianza al panamericanismo civilizador: los Estados Unidos y la Argentina, 1928-1946". (Manuscrito).

STEIL, Benn

2013 The Battle of Bretton Woods: John Maynard Keynes, Harry Dexter White, and the Making of a New World Order. Princeton. Princeton University Press.

Tulchin, Joseph

1971 The Aftermath of War: World War I and US Policy toward Latin America. New York. New York University Press.

U. S. Advisory Economic Mission to Venezuela

1940 Report to the Minister of Finance by the American Economic Mission to Venezuela. Washington.

WebBer, Carolyn - Wildavsky, Aaron

1986 A History of Taxation and Expenditure in the Western World. Nueva York. Simon \& Schuster.

WALTERS, F. P.

1971 Historia de la Sociedad de Naciones. Madrid. Tecnos.

Wood, Bryce

1985 The Dismantling of the Good Neighbor Policy. Austin. University of Texas Press. 